\title{
Direct Imaging of Fine Structures in Giant Planet Forming Regions of the Protoplanetary Disk around AB Aurigae ${ }^{1}$
}

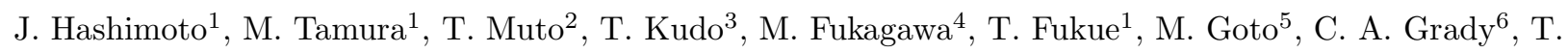 \\ Henning $^{5}$, K. Hodapp ${ }^{7}$, M. Honda ${ }^{8}$, S. Inutsuka ${ }^{9}$, E. Kokubo ${ }^{1}$, G. Knapp ${ }^{10}$, M. W. McElwain ${ }^{10}$, M. \\ Momose $^{11}$, N. Ohashi ${ }^{12}$, Y. K. Okamoto ${ }^{11}$, M. Takami ${ }^{12}$, E. L. Turner ${ }^{10,13}$, J. Wisniewski ${ }^{14}$, M. Janson ${ }^{15}$, \\ L. Abe ${ }^{16}$, W. Brandner ${ }^{5}$, J. Carson ${ }^{5,17}$, S. Egner ${ }^{3}$, M. Feldt ${ }^{5}$, T. Golota ${ }^{3}$, O. Guyon ${ }^{3}$, Y. Hayano ${ }^{3}$, M. \\ Hayashi $^{3}$, S. Hayashi ${ }^{3}$, M. Ishii ${ }^{3}$, R. Kandori ${ }^{1}$, N. Kusakabe ${ }^{1}$, T. Matsuo ${ }^{1}$, S. Mayama ${ }^{18}$, S. Miyama ${ }^{1}$, J.-I. \\ Morino $^{1}$, A. Moro-Martin ${ }^{19,10}$, T. Nishimura ${ }^{3}$, T.-S. Pyo ${ }^{3}$, H. Suto ${ }^{1}$, R. Suzuki ${ }^{20}$, N. Takato ${ }^{3}$, H. Terada ${ }^{3}$,

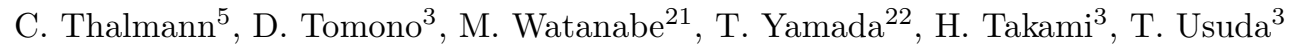

\begin{abstract}
We report high-resolution $1.6 \mu \mathrm{m}$ polarized intensity $(P I)$ images of the circumstellar disk around the Herbig Ae star AB Aur at a radial distance of 22 AU (0."15) up to 554 AU (3."85),
\end{abstract}

\footnotetext{
${ }^{1}$ National Astronomical Observatory of Japan, 2-21-1 Osawa, Mitaka, Tokyo 181-8588, Japan; jun.hashimoto@nao.ac.jp, motohide.tamura@nao.ac.jp

${ }^{2}$ Tokyo Institute of Technology, 2-12-1 Ookayama, Meguro, Tokyo 152-8551, Japan

${ }^{3}$ Subaru Telescope, 650 North A'ohoku Place, Hilo, HI 96720, USA

${ }^{4}$ Osaka University, 1-1, Machikaneyama, Toyonaka, Osaka 560-0043, Japan

${ }^{5}$ Max Planck Institute for Astronomy, Heidelberg, Germany

${ }^{6}$ Eureka Scientific and Goddard Space Flight Center, Greenbelt, MD 20771, USA

${ }^{7}$ University of Hawaii,640 North A'ohoku Place, Hilo, HI 96720, USA

${ }^{8}$ Kanagawa University, 2946 Tsuchiya, Hiratsuka, Kanagawa 259-1293, Japan

${ }^{9}$ Nagoya University, Furo-cho, Chikusa-ku, Nagoya, Aichi 464-8602, Japan

${ }^{10}$ Department of Astrophysical Sciences, Princeton University, NJ 08544, USA

${ }^{11}$ Ibaraki University, 2-1-1 Bunkyo, Mito, Ibaraki 310-8512, Japan

${ }^{12}$ Institute of Astronomy and Astrophysics, Academia Sinica, P.O. Box 23-141, Taipei 10617, Taiwan

${ }^{13}$ Institute for the Physics and Mathematics of the Universe, The University of Tokyo, Kashiwa 227-8568, Japan

${ }^{14}$ University of Washington, Seattle, Washington, USA

${ }^{15}$ University of Toronto, Toronto, Canada

${ }^{16}$ Laboratoire Hippolyte Fizeau, UMR6525, Universite de Nice Sophia-Antipolis, 28, avenue Valrose, 06108 Nice Cedex 02 , France

${ }^{17}$ Department of Physics and Astronomy, College of Charleston, 58 Coming St., Charleston, SC29424, USA

${ }^{18}$ The Graduate University for Advanced Studies, Shonan International Village, Hayama-cho, Miura-gun, Kanagawa 240-0193, Japan

${ }^{19}$ Department of Astrophysics, CAB - CSIC/INTA, 28850 Torrej'on de Ardoz, Madrid, Spain

${ }^{20}$ TMT Observatory Corporation, 1111 South Arroyo Parkway, Pasadena, CA 91105 USA

${ }^{21}$ Department of Cosmosciences, Hokkaido University, Sapporo 060-0810, Japan

${ }^{22}$ Astronomical Institute, Tohoku University, Aoba, Sendai 980-8578, Japan
} 
which have been obtained by the high-contrast instrument HiCIAO with the dual-beam polarimetry. We revealed complicated and asymmetrical structures in the inner part ( $\lesssim 140 \mathrm{AU})$ of the disk, while confirming the previously reported outer $(r \gtrsim 200$ AU) spiral structure. We have imaged a double ring structure at $\sim 40$ and $\sim 100 \mathrm{AU}$ and a ring-like gap between the two. We found a significant discrepancy of inclination angles between two rings, which may indicate that the disk of AB Aur is warped. Furthermore, we found seven dips (the typical size is $\sim 45$ AU or less) within two rings as well as three prominent $P I$ peaks at $\sim 40 \mathrm{AU}$. The observed structures, including a bumpy double ring, a ring-like gap, and a warped disk in the innermost regions, provide essential information for understanding the formation mechanism of recently detected wide-orbit $(r>20 \mathrm{AU})$ planets.

Subject headings: planetary systems — protoplanetary disks — stars: individual (AB Aurigae) — stars: pre-main sequence - polarization

\section{Introduction}

Circumstellar disks are usually formed around young stars and are intricately tied to the origin of planets (Shu et al. 1987; Beckwith \& Sargent 1996). Giant planets have been considered to form via gas accretion onto rocky cores in such disks (e.g., Pollack et al. 1996), which can successfully explain "normal" giant planets like ours. However, recent direct detections of companions with masses of a few up to a few tens of $M_{\mathrm{J}}$ at distances $>20 \mathrm{AU}$, beyond what had been thought to be the planet forming zone (Marois et al. 2008; Kalas et al. 2008; Thalmann et al. 2009), pose a challenge for the standard core-accretion scenario where planets are formed in-situ. Planet migration after formation such as planet-planet scattering (e.g., Veras et al. 2009), or gravitational instability (GI) scenarios (e.g., Durisen et al. 2007; Inutsuka et al. 2010) may account for such wide-orbit massive planets. To test these theories, information on the detailed structures of the inner $(r<50 \mathrm{AU})$ regions of protoplanetary disks is crucial.

One of the best candidates to investigate the inner $(r<50 \mathrm{AU})$ regions is the prototype young intermediatemass star AB Aur which is one of the most intensively studied Herbig Ae stars $\left(d=144_{-17}^{+23} \mathrm{pc} ; M=2.4 \pm 0.2\right.$ $M_{\odot}$; an age of $4 \pm 1 \mathrm{Myr}$; van den Ancker et al. 1997; DeWarf et al. 2003). AB Aur is also known to possess a large $(r>1000 \mathrm{AU})$ nebula (Grady et al. 1999). A more compact $(r \sim 450 \mathrm{AU})$ rotating disk was detected by millimeter observations with a disk mass of $\sim 20 M_{\mathrm{J}}$ (Henning et al. 1998). Near-infrared imaging has revealed a spiral structure in the outer $(r \gtrsim 200 \mathrm{AU})$ part of the disk (Fukagawa et al. 2004). A more recent study revealed structures at intermediate distances from the star (40 AU $<r<300 \mathrm{AU}$ ) and reported a possible point source in a "dip" of the PI image in the inner disk region (Oppenheimer et al. 2008). On the other hand, a subsequent study failed to confirm the point source (Perrin et al. 2009). However, all these observations were limited either by the inner working distance $(r>40 \mathrm{AU})$ or the spatial resolution $(\sim 14$ AU). In this Letter, we present polarized intensity (hereafter $P I$ ) images with the smallest inner working angle to the central star $(r>22 \mathrm{AU})$ and that the highest spatial resolution (9 AU) to date to reveal fine structures in the inner $(r<50 \mathrm{AU})$ regions.

\footnotetext{
${ }^{1}$ Based on data collected at the Subaru Telescope, which is operated by the National Astronomical Observatory of Japan.
} 


\section{Observations \& Data Reduction}

$H$-band $(1.6 \mu \mathrm{m})$ linear polarization images of $\mathrm{AB}$ Aur were obtained with a newly commissioned high-contrast imaging instrument HiCIAO (Tamura et al. 2006) combined with dual-beam polarimetry on the Subaru 8.2m Telescope on 2009 October 31 UT. The observations were conducted under the program SEEDS (Strategic Explorations of Exoplanets and Disks with Subaru; Tamura 2009). In the polarimetric observation mode, $o$ - and $e$-rays are observed simultaneously, each has $10^{\prime \prime}$ by $20^{\prime \prime}$ field of view with a pixel scale of 9.3 mas/pixel, and therefore, $P \mathrm{I}$ is free from subtraction of reference PSFs. For the AB Aur observations, we used a small circular occulting mask of $0 .{ }^{\prime \prime} 3$ diameter. The polarizations were measured by rotating the half waveplate to four angular positions (in the order of $0^{\circ}, 45^{\circ}, 22.5^{\circ}$, and $67.5^{\circ}$ ). We obtained seven data sets by repeating the cycle of waveplate rotations taking a 23.7-s exposure per waveplate position each cycle. The adaptive optics system (AO188; Havano et al. 2004) provided a diffraction limited and mostly stable stellar PSF with FWHM of $0 .{ }^{\prime \prime} 06$ in the $H$ band. Low quality images were removed prior to final production of the images. We used the four best data sets and the total integration time of the $P I$ image was $189.6 \mathrm{~s}$.

The data were reduced in the standard manner of infrared image reduction using IRAF2, namely subtracting a dark frame and dividing by a flat frame. The Stokes $Q$ and $U$ parameters were created by subtracting two split images, in the standard approach for differential polarimetry (e.g., Hinklev et al. 2009). Then, we calculated $P I$ as $\sqrt{Q^{2}+U^{2}}$ shown in figures 1 and 2, $P I$ images have been used by many researchers (e.g., Close et al. 1997; Oppenheimer et al. 2008) as they have a significant advantage over the total intensity (hereafter $I$ ) images due to difficulties with accurate reference PSF subtractions.

Since our focus is the $P I$ rather than $I$ image of AB Aur, it is first essential to demonstrate that the $P I$ around $\mathrm{AB}$ Aur does not produce any artificial structures. For this purpose, the polarization vector pattern, and its azimuthal dependence is a good indicator of whether $P I$ is affected by the bright central star. We calculated averaged azimuthal polarization profiles from a reference PSF-subtracted $I$ image, using HD 282411 as the PSF reference star. The reference star HD 282411 was observed just after the observations of $\mathrm{AB}$ Aur and was observed in the same observation mode as AB Aur. In the PSF subtraction process, we searched for the best subtracted images in the various star-PSF frames while varying the registration and scaling to minimize residuals.

Fig 3 shows the observed polarization vector (the position angle as $0.5 \arctan (U / Q)$ ) image of AB Aur (see the caption of Fig 3 about the construction of the vector image) and a histogram of the angles between the polarization vectors and lines from the stellar position to the vector position. The polarization vector pattern is a good indicator of whether the Stokes $Q$ and $U$ are affected by residual speckle noise of the bright central star. This is because when the Stokes $Q$ and $U$ contain such noise, the polarization vectors show either random or parallel alignment. As a result of Gaussian fitting in the histogram, we found that the central position and FWHM are $90.1^{\circ} \pm 0.2^{\circ}$ and $4.3^{\circ} \pm 0.4^{\circ}$, respectively. Since the polarization vectors are clearly centrosymmetric, we conclude that the residual speckle noise of AB Aur is quite low and any features identified in our PI images (the ring gap, dips, and peaks) are real.

\footnotetext{
2 IRAF is distributed by the National Optical Astronomy Observatories, which are operated by the Association of Universities for Research in Astronomy, Inc., under cooperative agreement with the National Science Foundation.
} 


\section{Results \& Discussion}

Fig[1 shows the PI image of AB Aur covering a radial distance of 22-554 AU (0."15-3."85). Before discussing the inner region, we compare the outer $(r \gtrsim 140 \mathrm{AU})$ structures of our PI image and the $I$ image of previous observations (Fukagawa et al. 2004). The morphology of the outer parts of these images is consistent at scales larger than the spatial resolution, although one is a $P I$ image and the other is an $I$ image. These images trace the scattered light of the central star by dust grains near the surface of the disk because the disk is optically thick at infrared wavelengths. Note, however, that the optically thin sub-millimeter continuum radiation also reveals some of the arms; the spirals are not simply surface corrugation but also reflect the structures near the midplane of the disk (Lin et al. 2006; Piétu et al. 2005).

The real advantage of our new observations is that they allow investigation of the inner $(r<50 \mathrm{AU})$ structure, comparable to our Solar-System. Fig 2 shows a magnified PI image of the inner part of AB Aur and the associated azimuthal profiles. The inner regions of the disk exhibit complex, non-axisymmetric morphological structures. We summarize the significant features revealed in our PI images as follows:

(a) We found two ellipse rings, as denoted in Fig 2 Table 1 shows the results of ellipse fitting. The position angles of the minor axes of these two rings are roughly consistent with the disk rotation axis inferred from CO emission line kinematics $\left(330^{\circ}\right.$; Piétu et al. 2005). We consider the ellipse shape to be due to inclination (Lagage et al. 2006); the south-east side of the ring is inclined towards us (Fukagawa et al. 2004). The difference of the inclination angles between the inner and outer rings suggests that the inner ring might be warped relative to the outer ring. In addition, we measured an offset of $0 . .^{\prime \prime} 19 \pm 0 . .^{\prime \prime} 01$ between the geometric center of the outer ring and the central star. However, since the direction of this offset at the far side of the disk with $313.6^{\circ} \pm 5.1^{\circ}$ is roughly consistent with the disk rotation axis, this offset is most likely due to a geometric effect of the flared disk (e.g., Fukagawa et al. 2004). Our detected double ring structure with a warp has not been reported in previous studies of AB Aur (Oppenheimer et al. 2008; Perrin et al. 2009).

(b) We found a wide ellipse ring-like gap between the two rings, as indicated in Fig 2 (hereafter the ring gap). The ellipse fitting results are also shown in table1 This ring gap is barely seen in previous PI images (Oppenheimer et al. 2008; Perrin et al. 2009). The far-side wall of the ring gap probably corresponds to the wall-like structure at $88 \mathrm{AU}$ inferred by mid-infrared observations (Honda et al. 2010). Another example of a gap is HD 100546 (Bouwman et al. 2003), in which they inferred that this Herbig Be star has a disk gap around $10 \mathrm{AU}$ based on the infrared SED. Interestingly, they also argued that AB Aur has a prototype disk without a gap in the inner region.

(c) We found in total seven small dips in the $P I$ within the two rings, which we refer to as Dip A to G as shown in Fig 2 The typical size of the dips is $\sim 45 \mathrm{AU}$ or less. The most prominent, Dip A at $\sim 100 \mathrm{AU}$, is consistent with those reported in the $P I$ images of previous studies (Oppenheimer et al. 2008; Perrin et al. 2009). Dip A is confirmed at the $3 \sigma$ confidence level in our $I$ image whose averaged azimuthal profile is shown in Figs 2. The detection of Dip A in our $I$ image shows that the $P I$ traces the $I$ pattern, and therefore, it is not solely a geometrical polarization effect as claimed (Perrin et al. 2009). This detection also suggests that the PI image can be used to discuss even in the inner regions where the $I$ image is affected by speckle noise. This is especially true when discussing the "local" structures rather than the "global" structures that can be affected by the inclination of the disk. In addition, we found three PI peaks in close vicinity to the occulting mask, which we refer to as P1 to P3 in Fig 2, The peaks in the outer rings seen in our PI image are not labeled here for simplicity. All the peak structures are extended and thus not point sources. 
(d) No point-like sources are detected in Dip A in either the PI or $I$ images, as opposed to Oppenheimer et al. (2008). Our observations are consistent with Perrin et al. (2009). When we assume that a companion is 100 $\%$ polarized in the $P I$ image, which is the faintest case as Oppenheimer et al. (2008), the upper limits of its mass at $5 \sigma$ (the absolute magnitude of 11.7 at the $H$ band) of the photon noise in Dip A are 5 and $6 M_{\mathrm{J}}$ for an age of 3 and $5 \mathrm{Myr}$, respectively (Baraffe et al. 2003). These derived upper limits of the masses are consistent with that of $1 M_{\mathrm{J}}$ inferred by the numerical simulations (Jang-Condell \& Kuchner 2010). On the other hand, our upper limits for point sources in the dips seen in the inner ring are 7 and $9 M_{\mathrm{J}}$ for these ages due to higher photon noise.

The structures of AB Aur's inner (22-120 AU) disk surface described above indicate that the disk is in an active and probably early phase of global evolution, and possibly one or more unseen planets are being formed in the disk.

One possible explanation for the non-axisymmetric structures is GI of the disk (e.g., Durisen et al. 2007). If Toomre's $Q$-parameter (defined as $Q=c_{\mathrm{s}} \kappa / \pi \mathrm{G} \Sigma$, where $c_{\mathrm{s}}, \kappa$, and $\Sigma$ are the sound speed, epicycle frequency, and surface density, respectively) is of the order of unity, GI occurs and a mode with a small number of arms is excited, that is, a pattern of the surface density arises that may resemble what we have observed. However, this GI possibility may be rejected for AB Aur (at present) because optically thin submillimeter observations indicate that Toomre's $Q$-parameter is of the order of ten (Piétu et al. 2005). It may be noted that the disk mass estimate from sub-mm emission has large uncertainties arising from the uncertainties in the optical properties of the dust particles.

The presence of unseen planets in the disk can also result in perturbations which extend over the disk scale even in the absence of GI. A low-mass planet in a disk excites a spiral density wave that co-rotates with the planet (Goldreich \& Tremaine 1979; Tanaka et al. 2002), while a high-mass planet opens a gap in addition to the excitation of a spiral (Lin \& Papaloizou 1986), thereby inducing a more significant, globally extended perturbation in the disk (see Papaloizou et al. 2007, for the review of disk-planet interaction). The gap opens when the amplitude of the perturbation caused by the embedded planet exceeds the order of unity. Since the amplitude of the perturbation scales with $q / h^{3}$, where $q$ is the mass ratio between the planet and the central star and $h=H / r$ is the disk aspect ratio (Tanaka et al. 2002), a crude estimate of the gap-opening mass is $q>h^{3}$. For the disk around AB Aur, the temperature of the disk at the location of the ring gap $(\sim 80 \mathrm{AU})$ is $20-30 K$ (Piétu et al. 2005; Lin et al. 2006), and therefore, the disk aspect ratio is expected to be $\sim 0.1$. Therefore, a planet with a mass of only $\sim 1 M_{\mathrm{J}}$ (consistent with the fact that we detected no point sources) situated in the ring gap in the vicinity of Dip A can form a gap at these distances. Such a planet cannot be directly seen if it is embedded in the disk equatorial plane; however, its perturbation can induce the observed structures such as the ring gap and the largest Dip A and is seen as "shadows" (Jang-Condell \& Kuchner 2010), while other peaks and small dips might be due to small perturbations. Furthermore, a warp in the inner region may be explained by the gravitational perturbation from unseen planets (Mouillet et al. 1997). It is also noted that, there is a possibility that the inner ring is intrinsically elliptical due to the influence of an unseen gravitating object, which could be another indication of the presence of a planet (Kley \& Dirksen 2006). From the present data, it is rather difficult to distinguish the cause of the elliptical shape of the inner ring: either a warped circular ring or an intrinsically elliptical ring. However, we consider that both possibility may be accounted for by at least one gravitating object embedded in a disk.

The perturbation caused by an embedded planet generally tends to co-rotate with the planet, and therefore the deviation of the pattern speed from the local rotation speed would be smoking-gun proof of 
the existence of the planet. The pattern speed of the spiral structure is given by

$$
\omega=0.78\left(\frac{\mathrm{M}}{2.4 \mathrm{M}_{\odot}}\right)^{\frac{1}{2}}\left(\frac{r_{\mathrm{p}}}{80 \mathrm{AU}}\right)^{-\frac{3}{2}}[\mathrm{deg} / \mathrm{yr}]
$$

where $M$ is the mass of the central star and $r_{\mathrm{p}}$ is the orbital radius of the planet. Such time variability can be observed for the next several years. We note that the existence of a planet in the AB Aur system, whose age is only 3-5 Myr, may pose a unique constraint on the planet-formation timescale because their formation via gas accretion have been considered to take about 10 Myr (e.g., Pollack et al. 1996).

Another intriguing explanation for the observed structure is magneto-rotational instability (MRI) (Balbus \& Hawley 1998). Although global numerical simulations of MRI is numerically challenging, some MRI calculations show that perturbation may extend over the disk (Steinacker \& Papaloizou 2002). It is also shown that MRI drives the disk wind which causes a significant perturbation at the disk surface (Suzuki et al. 2010). In this case, the timescale of variability is on the order of the local rotation timescale, which is longer than that caused by the inner unseen planet.

In summary, the fine structures including the double ring structure with a warp as well as the ring gap detected by our observations most likely have an origin in planetary perturbation, but GI or MRI can also be a promising cause of the detailed structure. A key future investigation would be the detection of the time variability of the structures, which can provide clues for understanding the formation mechanisms of the wide-orbit companions discovered by direct imaging observations around A stars (Marois et al. 2008; Kalas et al. 2008) and a G star (Thalmann et al. 2009; Janson et al. 2010) as well as a number of physical processes ongoing in the active protoplanetary disk.

We are grateful to an anonymous referee for providing many useful comments leading to an improved paper. This work is partly supported by a Grant-in-Aid for Science Research in a Priority Area from MEXT and by the Mitsubishi Foundation. We also acknowledge support from AST-1008440 (CAG), AST-1009314 (JPW), and a Chretien International Research Grant (JPW). 
Table 1: Observational results of AB Aurigae ${ }^{a}$

\begin{tabular}{cccc}
\hline \hline & Outer ring & Ring gap & Inner ring \\
\hline Diameter of the major axis (AU) & $210.8 \pm 2.5$ & $170.2 \pm 2.0$ & $92.0 \pm 6.8$ \\
Diameter of the minor axis (AU) & $188.1 \pm 2.2$ & $149.1 \pm 1.7$ & $67.2 \pm 4.2$ \\
Position angle of the major axis $\left(^{\circ}\right)$ & $36.6 \pm 6.6$ & $36.2 \pm 5.2$ & $64.6 \pm 8.9$ \\
Inclination $^{\mathrm{b}}\left(^{\circ}\right)$ & $26.8 \pm 1.9$ & $28.8 \pm 1.7$ & $43.1 \pm 6.8$ \\
Geometric center $^{\mathrm{c}}(\mathrm{mas}:$ mas) & $(121 \pm 7: 127 \pm 8)$ & $(92 \pm 4: 40 \pm 7)$ & $(54 \pm 17: 4 \pm 13)$ \\
Width $^{\mathrm{d}}(\mathrm{AU})$ & $29.8 \pm 1.3$ & $16.1 \pm 1.6$ & $32.1 \pm 2.5$ \\
\hline
\end{tabular}

${ }^{a}$ In the ellipse fitting for the two rings and ring gap, the peak and bottom positions were first directly determined by the averaged radial profile at position angles every $5^{\circ}$ and $15^{\circ}$ (corresponding to our spatial resolution) for the two rings and the ring gap, respectively. We then conducted an ellipse fit using an implementation of the nonlinear least-squares Marquardt-Levenberg algorithm with five free parameters of lengths for the major and minor axes, position angle, and central positions.

${ }^{b}$ Derived from the ratio of the major and minor axes.

${ }^{c}$ Central position $(0,0)$ is corresponding to the stellar position.

${ }^{d}$ Assuming circular structures with given inclinations. The values of the full width at half maximum are derived from Gaussian fitting of the radial profile at the given position angle of the major axis in the north east part. 

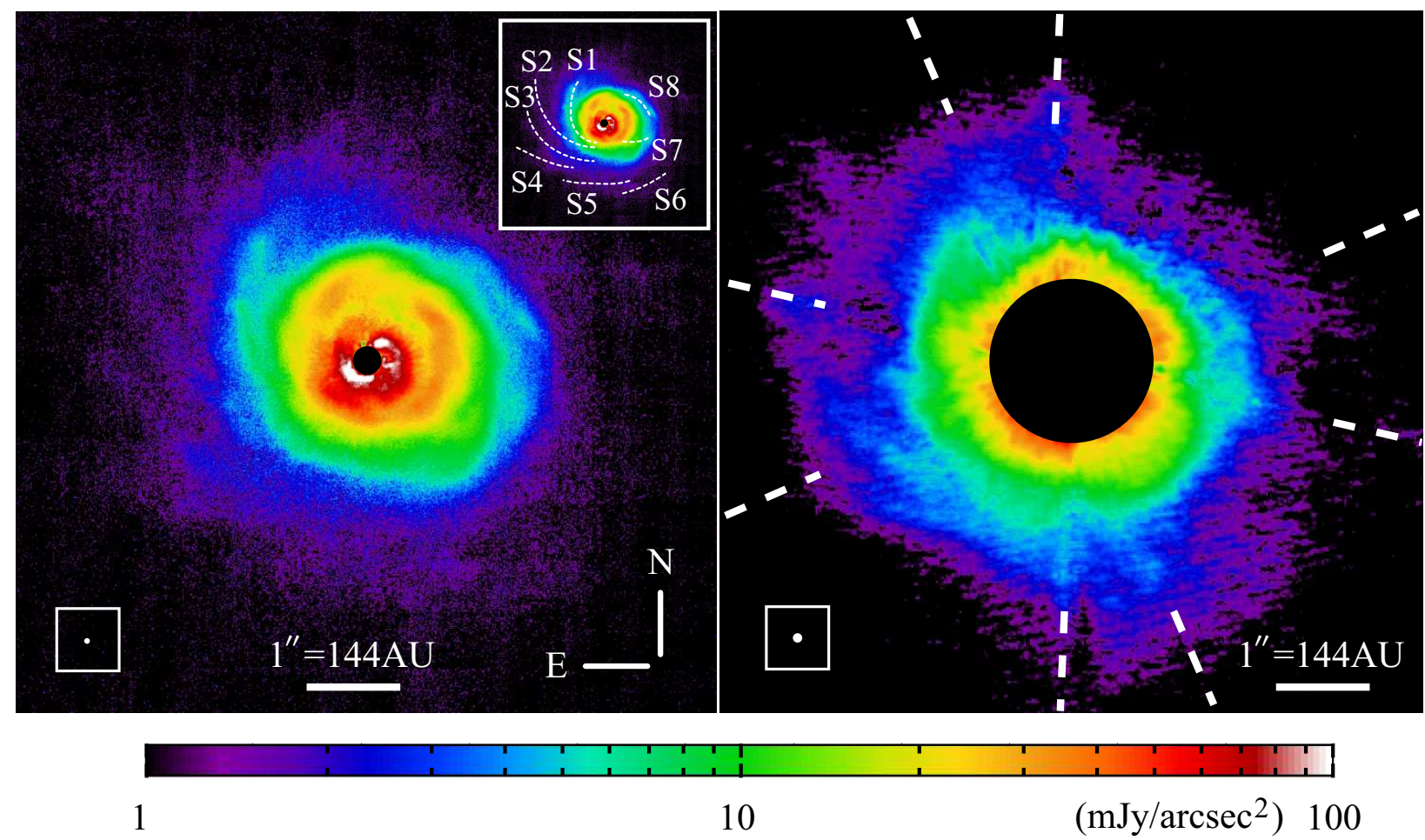

Fig. 1.- Near-infrared ( $H$ band) $P I$ and $I$ images of AB Aur. Left: The HiCIAO PI image with a coronagraphic occulting mask of $0 . " 3$ diameter. The multiple spiral structures we refer to as S1 to S8 are identified in the top-right inset, of which S7 and S8 are newly found. Right: The CIAO reference PSFsubtracted $I$ image with a software mask of $1 . .^{\prime \prime} 7$ diameter (Fukagawa et al. 2004). In contrast to the original image, this image shown here is not divided by the square of the radial distance from the star. The field of view in both images is $7 . .^{\prime \prime} 5$ by $7 .{ }^{\prime \prime} 5$. The solid circles in the left-bottom inset in both images represent the spatial resolution of $0 .{ }^{\prime \prime} 06$ and $0 .{ }^{\prime \prime} 1$, respectively. 
AU
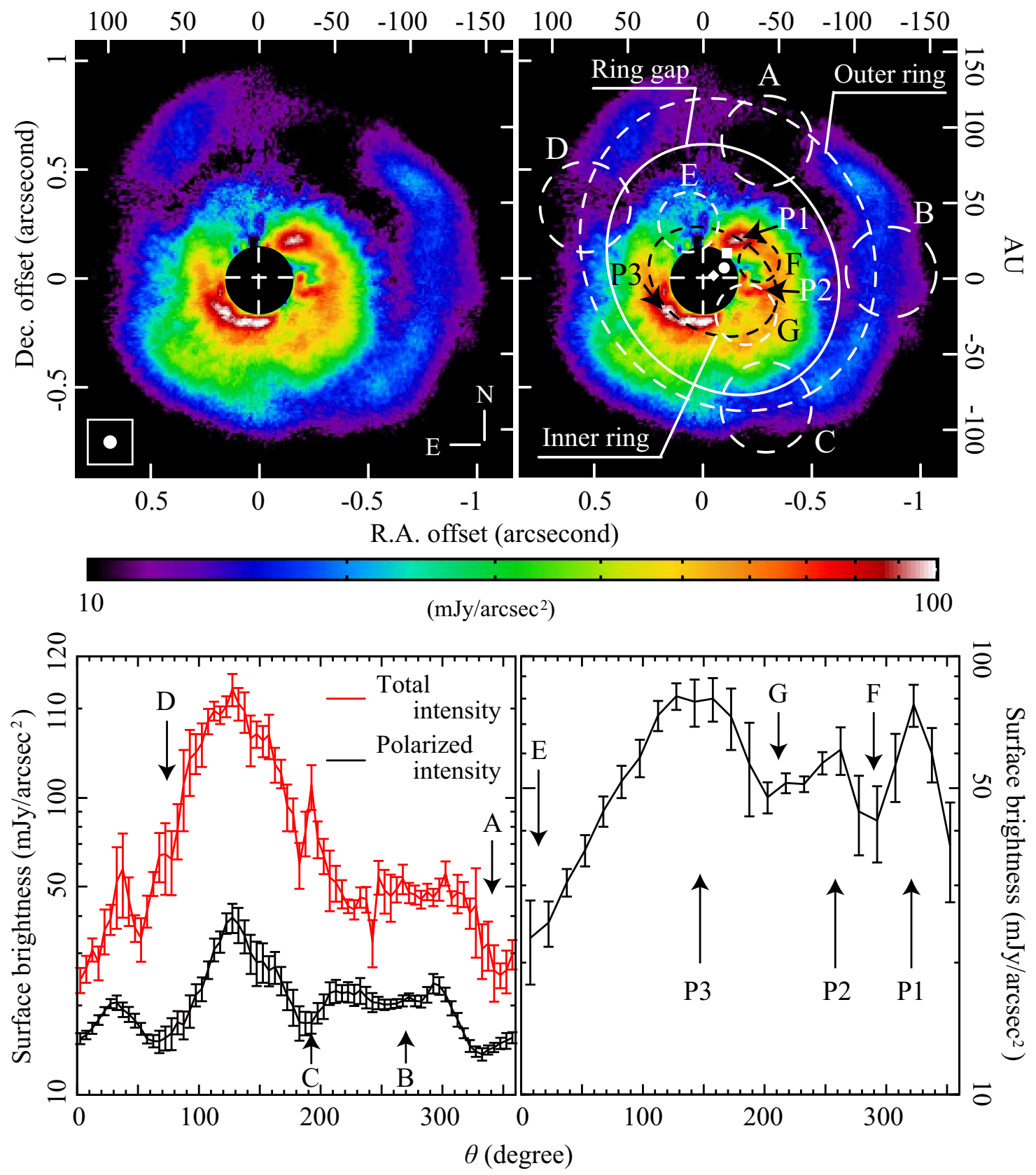

Fig. 2.- Magnified view of the inner $P I$ images of $\mathrm{AB}$ Aur and their averaged azimuthal profiles. Top: magnified $P I$ image with a coronagraphic occulting mask of $0 .{ }^{\prime \prime} 3$ diameter (left) and the features of the $P I$ image (right). Central position $(0,0)$ is the stellar position. The outer and inner rings are denoted by the dashed ellipsoids. The solid ellipsoid indicates the wide ring gap. The dashed circles (A to G) represent small dips in the two rings. The filled diamond, circle, and square represent the geometric center of the inner ring, ring gap, and outer ring, respectively. The field of view in both images is $2 .^{\prime \prime} 0$ by $2 .{ }^{\prime \prime} 0$. The solid circle in the left-bottom inset represents the spatial resolution of 0."06. Bottom-left: Averaged azimuthal profiles of the outer ring for the $P I$ (black) and reference PSF-subtracted $I$ (red) images. The profile is averaged every $5^{\circ}$ in position angle (corresponding to resolution) in the outer ring. Bottom-right: Same with the bottom-left image, but for the inner ring with every $15^{\circ}$ in position angle (corresponding to resolution) in the inner ring. 

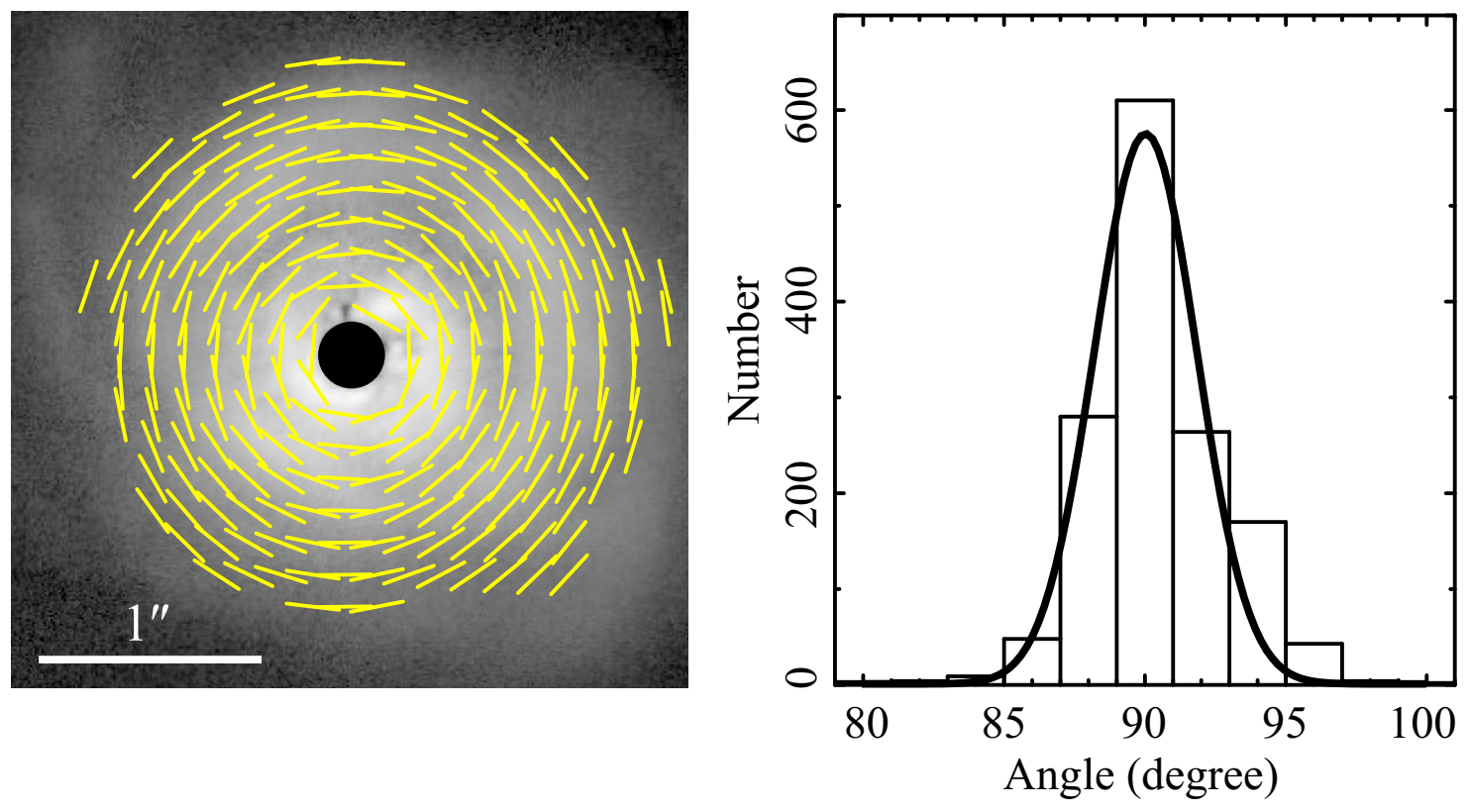

Fig. 3.- Polarization vector pattern of $\mathrm{AB}$ Aur. Left: Polarization vectors superposed on the $P I$ image of $\mathrm{AB}$ Aur in the $H$ band. The plotted vectors are based on $6 \times 6$ pixel binning which corresponds to the spatial resolution, and have a polarized intensity larger than $50 \sigma$. Not all the vectors are plotted and their lengths are not to scale for the purposes of presentation. Right: Histogram of angles between polarization vectors and lines from the mask center to the vector position. As a result of Gaussian fitting, the central position and FWHM are $90.1^{\circ} \pm 0.2^{\circ}$ and $4.3^{\circ} \pm 0.4^{\circ}$, respectively. 


\section{REFERENCES}

Balbus, S. A., \& Hawley, J. F. 1998, Rev. Mod. Phys. 70, 1

Baraffe, I. Chabrier, G., Barman, T. S., Allard, F., \& Hauschildt, P. H. 2003, A\&A, 402, 701

Beckwith, S. V. W., \& Sargent, A. I. 1996, Nature, 383, 139

Bouwman, J., de Koter, A., Dominik, C., \& Waters, L. B. F. M. 2003 A\&A, 401, 577

Close, L. M. et al. 1997, ApJ, 489, 210

DeWarf, L. E., Sepinsky, J. F., Guinan, E. F., Ribas, I., \& Nadalin, I. 2003, ApJ, 590, 357

Durisen, R. H., Boss, A. P., Mayer, L., Nelson, A. F., Quinn, T., \& Rice, W. K. M. 2007, in Protostars and Planets V, ed. B. Reipurth, D. Jewitt, \& K. Keil (Tucson: Univ. Arizona Press), 607

Fukagawa, M. et al. 2004, ApJ, 605, L53

Goldreich, P., \& Tremaine, S. 1979, ApJ, 233, 857

Grady, C. A. et al. 1999, ApJ, 523, L151

Hayano, Y. et al. 2004, Proc. SPIE, 5490, 1088

Henning, Th., Burkert, A., Launhardt, R., Leinert, Ch., \& Stecklum, B. 1998, A\&A, 336, 565

Hinkley, S. et al. 2009, ApJ, 701, 804

Honda, M. et al. 2010, ApJ, 718, L199

Inutsuka, S.-i., Machida, M., \& Matsumoto, T. 2010, ApJ, 718, L58

Jang-Condell, H., \& Kuchner, M. J. 2010, ApJ, 714, L142

Janson, M. et al. 2010, in press, arXiv:1011.5505

Kalas, P. et al. 2008, Science 322, 1345

Kley, W., \& Dirksen, G. 2006,A\&A, 447, 369

Lagage, P. O. et al. 2006, Science, 314, 621

Lin, D. N. C., \& Papaloizou, J. 1986, ApJ, 307, 395

Lin, S., Ohashi, N., Lim, J., Ho, P. T. P., Fukagawa, M., \& Tamura, M. 2006, ApJ, 645, 1297

Marois, C. et al. 2008, Science, 322, 1348

Mouillet, D., Larwood, J. D., Papaloizou, J. C. B., \& Lagrange, A. M. 1997, MNRAS, 292, 896

Oppenheimer, B. R. et al. 2008, ApJ, 679, 1574

Papaloizou, J. C. B., Nelson, R. P., Kley, W., Masset, F. S., \& Artymowicz, P. 2007, in Protostars and Planets V, ed. B. Reipurth, D. Jewitt, \& K. Keil (Tucson: Univ. Arizona Press), 655 
Perrin, M. D., Schneider, G., Duchene, G., Pinte, C., Grady, C. A., Wisniewski, J. P., \& Hines, D. C. 2009, ApJ, 707, L132

Piétu, V., Guilloteau, S., \& Dutrey, A. 2005, A\&A, 443, 945

Pollack, J. B., Hubickyj, O., Bodenheimer, P., Lissauer, J. L., Podolak, M., \& Greenzweig, Y. 1996, Icarus, 124,62

Shu, F. H., Adams, F. C., \& Lizano, S. 1987, ARA\&A, 25, 23

Steinacker, A., \& Papaloizou, J. C. B. 2002, ApJ, 571, 413

Suzuki, T. K., Muto, T., \& Inutsuka, S.-i. 2010, ApJ, 718, 1289

Tamura, M. et al. 2006, Proc. SPIE, 6269, 28

Tamura, M. 2009, in proc. "Exoplanets and Disks: Their Formation and Diversity" (2009 March 9-12), eds. Usuda, T., Ishii, M. and Tamura, M. (National Astronomical Observatory of Japan), p. 11-16

Tanaka, H., Takeuchi, T., \& Ward, W. R. 2002, ApJ, 565, 1257

Thalmann, C. et al. 2009, ApJ, 707, L123

van den Ancker, M. E., The, P. S., Tjin A Djie, H. R. E., Catala, C., de Winter, D., Blondel, P. F. C., \& Waters, L. B. F. M. 1997, A\&A, 324, L33

Veras, D., Crepp, J. R., \& Ford, E. B. 2009, ApJ, 696, 1600 